\title{
INCLUSIÓN EDUCATIVA EN LA ESCUELA SECUNDARIA. UNA EXPERIENCIA EXITOSA
}

\author{
Inclusion at the Secondary Level. A Successful Experience
}

\author{
DELFINA GARINO*1 \\ http://orcid.org/0000-0002-0320-8830 \\ *IPEHCS-UNCo-CONICET. Argentina. \\ Correspondencia: delgarino@gmail.com
}

Recibido: 24-11- 2017

Revisado: 27-11-2017

Aceptado: 02-01-2018

Resumen: Aunque desde 2006 se estableció la obligatoriedad legal del nivel secundario completo en Argentina, se observan problemas vinculados a la retención y al desarrollo de procesos educativos significativos para quienes aprenden. El propósito de este artículo es analizar, desde una perspectiva metodológica cualitativa, la propuesta educativa de una escuela secundaria que ofrece formación para el trabajo. Se retoma la voz de sus egresados para examinar la inclusión de los jóvenes a la escuela secundaria (en términos de retención) y en la escuela secundaria (en términos de desarrollo de procesos de aprendizajes significativos). Entre los hallazgos se destaca la valoración del seguimiento personalizado y de la formación para el trabajo como estrategias que posibilitaron la inclusión educativa.

Palabras clave: escuela secundaria; inclusión educativa; formación para el trabajo; jóvenes; deserción.

\begin{abstract}
Compulsory secondary school was stablished by law in Argentina in 2006. However, there are still problems that refer to drop off and to the development of meaningful learning processes for students. From a qualitative analysis perspective, this article analyzes the educational proposal of a secondary school with vocational training and takes up the voice of its graduates to analyze the inclusion of young people "to" secondary school (in terms of retention) and "in" secondary school (in terms of developing meaningful learning processes for students). Youngsters assessed work formation and personal containment as strategies that had enabled their educational inclusion.
\end{abstract}

Keywords: secondary school; educational inclusion; vocational training; youth; drop out.

\footnotetext{
${ }^{1}$ Doctora en Ciencias Sociales de la Universidad de Buenos Aires (Argentina). Licenciada en Sociología. Becaria Postdoctoral en el Instituto Patagónico de Estudios de Humanidades y Ciencias Sociales-Universidad Nacional del Comahue-Consejo Nacional de Investigaciones Científicas y Técnicas (IPEHCS-UNCo-CONICET). Docente de la Universidad Nacional del Comahue (Argentina). Coordinadora del GT CLACSO "Juventudes e inclusión laboral", miembro del Programa de Estudios Sobre Juventud, Educación y Trabajo (PREJET-CIS-IDES-CONICET). Líneas de trabajo: trayectorias de formación para el trabajo en escuelas secundarias e inserción laboral de jóvenes, articulación educación secundaria-mundo del trabajo, formación e inserción laboral de jóvenes en el sector hidrocarburífero.
} 


\section{INTRODUCCIÓN}

En la actualidad, tanto en Argentina como en otros países de América Latina, los jóvenes enfrentan grandes dificultades para insertarse laboralmente, así como para culminar los niveles obligatorios de educación, fenómenos que muchas veces están interrelacionados.

Las causas de los vaivenes en los procesos de inserción laboral de jóvenes se deben, en parte, a la precariedad y segmentación del mercado de trabajo, lo cual implica que se pueden encontrar en el proceso productivo distintos segmentos laborales que ofrecen condiciones de trabajo y de ingresos diferenciadas (Bertranau y Casanova, 2014) ${ }^{2}$. Claro está que el grado de segmentación no es el mismo para todos los niveles de instrucción, sino que desciende conforme estos aumentan.

Además, los indicadores que refieren, por ejemplo, a la desocupación, a la informalidad y a la subocupación horaria, siempre presentan cifras más alarmantes para los jóvenes que para los adultos. Esta situación se agrava para quienes han salido tempranamente del sistema educativo (especialmente para quienes no han completado los niveles obligatorios de educación), pertenecen a los sectores con menos recursos de la población (Pérez, 2008) y para el caso de las mujeres jóvenes (Organización Internacional del Trabajo, 2013).

Respecto del sistema educativo formal, en el año 2006 Argentina estipuló la obligatoriedad legal del nivel secundario completo a través de la sanción de la Ley de Educación Nacional №26.206/06, ley que regula el sistema educativo ${ }^{3}$, acumulando entre 13 y 14 años de educación obligatoria según la modalidad que se cursa. Sin embargo, dado que esta obligatoriedad no modificó su racionalidad selectiva original (Tiramonti, 2011), es decir, el hecho de tener a los miembros de sectores sociales altos y medios como destinatarios principales, los jóvenes (especialmente aquellos de sectores sociales bajos) experimentan dificultades para permanecer en el sistema educativo y terminar el nivel. Cabe aclarar que esta tendencia a la expansión de la obligatoriedad del secundario, así como las dificultades referidas a la retención, no son específicas del caso argentino, sino que se expanden en varios países de América Latina (Cabrera Borges, 2017; Arriagada y López, 2015; Croso, 2009).

\footnotetext{
${ }^{2}$ La existencia de segmentos diferenciados dentro de la economía supone que es posible hallar distintos grupos de trabajadores en el mercado laboral, que varían según el tipo de contratación, estabilidad, remuneraciones y cumplimiento de derechos que perciben, tanto al comparar la economía formal con la informal, así como al interior de cada una de estas (Bertranau y Casanova, 2014).

${ }^{3}$ Establece que el Estado debe proveer la educación para todos los habitantes de la Nación; que el Estado Nacional fija la política educativa y que debe garantizar el financiamiento del sistema educativo; fija su estructura; establece que el Consejo Federal de Educación (organismo de concertación de la política educativa nacional) define los contenidos curriculares comunes y núcleos de aprendizaje prioritarios, mientras que las jurisdicciones y las instituciones educativas pueden establecer contenidos curriculares acordes a sus realidades sociales, culturales y productivas.
} 
En este contexto, se retoman planteos que reflexionan sobre los procesos de exclusión de los estudiantes de la escuela, pero también en la escuela (Gluz, 2016) y que habilitan al menos dos discusiones. Por un lado, las dificultades que encuentran los jóvenes para permanecer y culminar los niveles obligatorios de educación; por otro lado, la forma en que se desarrollan sus procesos de escolarización, y que supone analizar también la estigmatización de las instituciones a las que asisten los jóvenes y la obtención de credenciales educativas devaluadas.

En este contexto interesa discutir acerca de qué saberes se promueven, transmiten y circulan en las escuelas, y la manera en que estos se vuelven relevantes (o no) a los ojos de los estudiantes. En este sentido, postulamos que el trabajo en torno a saberes que aparecen como significativos para los jóvenes promueve formas de inclusión en la escuela, y retomamos a Puiggrós y Galiano (2004) cuando plantean que en tanto pueden incidir en la construcción de las subjetividades de quienes los aprenden, otorgar herramientas para transformar el entorno donde viven y afectar el curso de sus vidas, pueden ser considerados saberes socialmente productivos.

Dado que el nivel secundario suele ser el último que los jóvenes cursan o finalizan, y que muchos jóvenes se insertan en el mercado de trabajo de manera inmediata a su culminación o abandono (si no lo han hecho simultáneamente al cursado de este nivel o incluso durante la escolaridad primaria), cabe preguntarse el espacio que tiene la formación para el trabajo y los sentidos que los jóvenes construyen sobre sus experiencias educativas en general, así como acerca de las prácticas de formación para el trabajo por las que circulan, en particular, favoreciendo experiencias escolares relevantes para los jóvenes.

Se plantea como hipótesis de trabajo que hay instituciones que despliegan distintos tipos de estrategias para incluir a los jóvenes a las escuelas y que además, gracias a los saberes y a las prácticas pedagógicas que promueven (especialmente a la formación orientada al mundo laboral), en tanto son significativas para los estudiantes, se constituyen en saberes socialmente productivos y a la vez en maneras de incluir a los jóvenes en las escuelas.

A partir de esta hipótesis, este artículo pone el foco, por un lado, en las estrategias que, desde la visión de los egresados de una escuela secundaria, habían facilitado su permanencia y terminalidad del nivel, favoreciendo la inclusión a la escuela; y, por otro lado, en los sentidos que habían construido sobre sus procesos educativos, y más específicamente, sobre su participación en prácticas de formación para el trabajo, para analizar si estos saberes habían favorecido procesos educativos más significativos. Para el análisis se retoma el caso de una escuela secundaria que tiene a la formación para el trabajo desde la perspectiva de la economía social en el centro de su propuesta educativa y busca favorecer la inclusión educativa de jóvenes de bajos recursos y brindar herramientas para intervenir en sus trayectorias de inclusión laboral. 
Este artículo es parte de los resultados de mi tesis doctoral, una investigación cualitativa que se orientó a estudiar las trayectorias de inserción laboral de jóvenes de sectores bajos que habían egresado de dos escuelas secundarias de la ciudad de Neuquén (Patagonia argentina) y que prestó especial atención a la incidencia de la formación recibida en el secundario en dichos procesos ${ }^{4}$.

La selección de las escuelas, así como la de los egresados, fueron realizadas por muestreo cualitativo de casos, elegidos en función de criterios teóricos entre los que se destacaron las especificidades de sus propuestas de formación para el mundo del trabajo, la visión del mundo del trabajo que sostenían y la población que recibían (concretamente, provenientes de hogares de bajos recursos materiales). La selección de la cohorte 2009 de egresados, por su parte, se debió a que era el primer grupo de graduados de una de las escuelas elegidas, bajo el supuesto de que entrevistar jóvenes que hubieran tenido un tiempo prolongado luego de finalizar sus estudios secundarios permitiría indagar acerca de las particularidades de sus recorridos vitales posteriores, prestando especial atención a las dinámicas de sus procesos de inserción laboral y a los sentidos construidos sobre sus experiencias escolares ${ }^{5}$.

El estudio de trayectorias se enmarcó en el enfoque biográfico (Sautu, 1999), que a través de relatos de sujetos individuales busca reflexionar en torno al interjuego que existe entre la biografía de ese sujeto y el contexto sociohistórico del cual es parte. En este marco, el relato que un sujeto hace de su propio recorrido vital tiene un lugar central, aunque se considera que esta narración supone un recorte más o menos consciente, por parte del entrevistado, sobre los recuerdos de los acontecimientos en los que participó directa o indirectamente, así como la interpretación que realiza sobre estos sucesos, mediada por sus experiencias de vida posteriores (Lomsky-Feder citado en Sautu, 1999). Efectivamente, el relato del entrevistado es el resultado de las interpretaciones que hace en el presente de los hechos que vivió, a partir de su propia historia y de sus proyectos y perspectivas futuras en el momento en que se lo entrevista (Mallimaci y Giménez Béliveau, 2006). Por este motivo, los sentidos y valoraciones de los jóvenes entrevistados respecto de sus experiencias escolares y del impacto de ellas en sus trayectorias supone una reconstrucción mediada por experiencias vitales posteriores.

\footnotetext{
${ }^{4}$ El trabajo de campo se realizó entre 2011 y 2014. Se realizaron entrevistas a informantes clave (se entrevistaron 6 directores de nivel y de modalidades del Consejo Provincial de Educación de Neuquén), análisis de documentos (se analizaron proyectos educativos y planes de estudios de 91 instituciones de nivel secundario de la Provincia), entrevistas semiestructuradas a 3 directivos de las escuelas que conformaron la muestra y entrevistas en profundidad a 8 profesores y a 35 egresados de ambas instituciones.

${ }^{5}$ Cabe aclarar que, si bien el objetivo original era entrevistar al total de egresados de esa cohorte de ambas escuelas, varios jóvenes no pudieron ser localizados y otros no quisieron ser entrevistados por la investigadora (se logró entrevistar al $60 \%$ del total de egresados de ambas instituciones).
} 
Para la construcción de los análisis que se presentan en este artículo, se retoman entrevistas en profundidad a 17 egresados $^{6}$ (varones y mujeres, realizadas en el segundo semestre del año 2014) de la Escuela N, en las que se trabajó la incidencia de la escuela en general y de las prácticas de formación para el trabajo en particular en sus trayectorias educativo-laborales posteriores, así como la construcción de sentidos sobre la experiencia escolar.

El artículo se estructura en cuatro apartados: en el primero se presenta el abordaje teórico del escrito; en el segundo se describen las características principales de la propuesta educativa de la escuela seleccionada; en el tercero se analiza la valoración de las experiencias escolares por parte de egresados de la escuela; finalmente se presentan las conclusiones.

\section{EXCLUSIÓN DE LA ESCUELA Y EN LA ESCUELA: PROBLEMAS NO RESUELTOS A PESAR DE LA OBLIGATORIEDAD DEL NIVEL}

Desde sus orígenes, la misión de la escuela secundaria en Argentina se orientó a la conformación de la ciudadanía y cumplió con el ideal de una "formación general" preparatoria para el cursado de estudios universitarios, privilegiando un currículum enciclopedista y humanista y dejando ciertos saberes (como los vinculados al trabajo) en un segundo plano en las propuestas pedagógicas. Se consolidó una estratificación de los saberes en académicos y técnicos: los primeros orientados al desarrollo personal y a la ciencia, los segundos al mundo del trabajo (Camillioni, 2006; Southwell, 2011).

Esta estratificación de saberes fue acompañada por una racionalidad selectiva, en la que los miembros de la elite y de sectores medios fueron sus destinatarios principales (Tenti Fanfani, 2007; Tiramonti, 2011), excluyendo de las aulas a quienes no se adaptaban al formato escolar imperante: "esta función selectiva está en la base de la definición de su formato y es el fundamento de una cultura escolar que naturaliza la exclusión de aquellos que no pueden responder o adaptarse a las exigencias de la institución" (Tiramonti, 2011, pp. 21-22).

Sin embargo, a mediados del siglo XX, se inició la apertura de la escuela secundaria a otros sectores sociales, proceso que llevó a su masificación y que derivó en 2006 en la obligatoriedad del nivel secundario completo a través de la Ley de Educación Nacional №26.206/06. Esta obligatoriedad se sumó al mandato social que plantea que todos los jóvenes deben cursar estudios secundarios y que se verifica en el hecho de que el mercado de trabajo exige cada vez más el título secundario, más allá de los conocimientos que requiera la labor de que se trate (Tenti Fanfani, 2007).

\footnotetext{
${ }^{6}$ Los nombres de los jóvenes que aparecen en los fragmentos de las entrevistas son ficticios.
} 
La particularidad de este proceso de masificación del nivel secundario es que se realiza en un contexto de desigualdad social. En este marco, las desigualdades que atraviesan la estructura de la sociedad son replicadas y sostenidas en el ámbito educativo. Efectivamente, pese al discurso igualitario que ha caracterizado al sistema educativo argentino, históricamente ha sostenido formas de integración escolar diferenciadas que perpetúan y reproducen las desigualdades sociales. Además, la retórica meritocrática asociada al sistema, niega las posiciones desiguales en las que se ubican los sujetos, oculta el papel que este desempeña en la producción y reproducción de las desigualdades y ayuda a construir y naturalizar jerarquías entre sectores, en las que quienes quedan en los segmentos superiores están incluidos y los que están en los inferiores son excluidos directa o indirectamente del sistema escolar (Dussel, 2009).

En efecto, la reproducción de la desigualdad se manifiesta, por ejemplo, en el hecho de que, en 2010, quienes pertenecían a sectores sociales bajos cuadruplicaban la no asistencia a la escuela en comparación con quienes pertenecían a sectores medios altos. Se observa, entonces, la exclusión de la escuela como un problema que está ligado a la desigualdad social, según el cual quienes se ubican en los estratos sociales más bajos tienen mayores posibilidades de ser excluidos del sistema educativo formal (Gluz, 2016).

A la exclusión de la escuela, Gluz (2016) agrega la exclusión en la escuela, que habilita a reflexionar en torno a distintas maneras en que los jóvenes pueden estar dentro de las instituciones educativas. Este concepto invita a pensar las formas en que muchos jóvenes transitan sus procesos de escolarización, y que supone considerar situaciones de retraso escolar, de entradas y salidas del sistema educativo, pero también de procesos de escolaridad de baja intensidad (Kessler, 2004).

La noción de escolaridad de baja intensidad refiere a aquellos jóvenes que están inscriptos y asisten de manera más o menos regular a la escuela, pero desarrollan un vínculo débil con la experiencia escolar. Esto puede manifestarse a partir de un estar conflictivo o un estar pasivo (sin involucrarse en las propuestas educativas) dentro de la institución. Analizar las causas de esta baja intensidad supone examinar las características del sistema escolar y de las dinámicas educativas que se le proponen a los estudiantes, que se suman a las condiciones en las que los jóvenes realizan sus procesos de escolarización.

La ampliación del sistema educativo a nuevos sectores sociales, entonces, supone una incorporación diferenciada, ya que quienes acceden no están en igualdad de condiciones que aquellos que fueron incorporados con anterioridad. Varias dinámicas coadyuvan en la producción de esta situación.

Por un lado, la cultura social de los estudiantes no necesariamente se acopla ni con la cultura escolar ni con el currículum que las instituciones educativas pretenden llevar adelante. En efecto, mientras éste posee características tales como homogeneidad, sistematicidad, continuidad y coherencia, entre otras, las culturas que 
traen consigo los jóvenes aparecen, a los ojos del sistema educativo, como fragmentadas, diversas, múltiples y flexibles, por lo que ambas tienden a entrar en conflicto. Esta situación es especialmente compleja para aquellos jóvenes que provienen de hogares de bajos recursos (Tenti Fanfani, 2007).

Por otro lado, estos jóvenes no ingresan a las mismas instituciones que los sectores dominantes, sino que circulan por instituciones estigmatizadas y que otorgan credenciales educativas devaluadas (Grupo Viernes, 2008). Efectivamente, la adquisición de credenciales académicas es una de las principales estrategias de reproducción de los sectores sociales (Bourdieu, 1988), pero las titulaciones que obtienen quienes pertenecen a sectores bajos no suelen ser valoradas de la misma manera que aquellas que obtienen quienes se ubican en los estratos superiores de la estructura social.

Además, si bien la mayor cantidad de años de educación facilita la obtención de un empleo de mejor calidad e ingresos más altos, la ampliación de la matrícula escolar sin un correlato al menos equivalente en el incremento de los puestos de trabajo a que esas titulaciones habilitan, produce una devaluación de las credenciales educativas:

es posible considerar que una titulación tiene todas las posibilidades de haber sufrido una devaluación, ya que el aumento del número de poseedores de titulaciones académicas es más rápido que el aumento del número de puestos a los que estas titulaciones conducían al principio del periodo (Bourdieu, 1988, p. 153).

Se produce entonces un proceso de "exclusión incluyente": si bien se incluye a ciertos sectores sociales en el sistema educativo, esta inclusión es precaria, inestable y habilita procesos educativos de calidad inferior a los posibles para otros sectores sociales (Gentili, 2011). Esta situación conlleva que los jóvenes pertenecientes a los grupos recientemente incorporados al nivel desarrollen trayectorias escolares no encauzadas, que insumen más tiempo que el teóricamente necesario para completar el nivel y que muchas veces derivan en el abandono escolar (Terigi, 2007).

Ante esta situación, desde algunas instituciones educativas se desarrollan estrategias orientadas a retener a los estudiantes, que a su vez se encuadran en acuerdos federales que operan como lineamientos para el funcionamiento del sistema educativo nacional. Ejemplo de esto son las "Orientaciones para la organización pedagógica e institucional de la educación secundaria obligatoria", aprobadas en la Resolución del Consejo Federal de Educación № 93/09, en las que se plantea, entre otras cuestiones, que las escuelas secundarias deben construir, de manera progresiva, propuestas educativas con distintas características, entre las que se destaca:

Partiendo del reconocimiento de las trayectorias reales de los adolescentes y jóvenes, se debe incorporar a la propuesta educativa instancias de atención a situaciones y momentos particulares que marcan los recorridos de los estudiantes y demandan un trabajo 
específico por parte de los equipos de enseñanza. Estas instancias pueden incluir, entre otras propuestas, apoyos académicos y tutoriales, dedicados a la orientación sobre la experiencia escolar, o bien espacios de aprendizaje que conecten la escuela con el mundo social, cultural y productivo (Consejo Federal de Educación, 2009, pp. 4-5).

Además, se pautan propuestas de apoyo institucional a las trayectorias escolares, definidos como espacios orientados a "brindar oportunidades de aprendizaje adicionales a los estudiantes que lo requieran" (p. 12). La implementación de estos espacios puede suponer la generación de itinerarios diferenciados según las necesidades de cada alumno.

Entre las estrategias orientadas a la retención que se implementan, se destaca el seguimiento personalizado de los alumnos, lo cual implica concebir a la educación como un derecho y se materializa de distintas maneras. De una parte, se concreta a partir de la reiteración de explicaciones o la búsqueda de alternativas pedagógicas que involucren a los estudiantes en las clases, como condición necesaria para que los alumnos desarrollen sus procesos de aprendizaje (Montes y Ziegler, 2010). De otra parte, también se manifiesta en las relaciones de proximidad que los adultos de las escuelas (docentes, directivos, asesores, preceptores, porteros, entre otros) muchas veces entablan con los alumnos (Nóbile, 2011).

Ambos aspectos poseen una dimensión emocional, que supone la confianza que los profesores depositan en los jóvenes respecto de sus capacidades para aprender y que deriva, entonces, en esfuerzos por parte de los docentes en la planificación de actividades y estrategias pedagógicas novedosas y atractivas para los alumnos, así como en la repetición de las explicaciones cuando algún estudiante no las comprende (Nóbile, 2011). Además, implica el conocimiento por parte de los adultos de las historias personales de los jóvenes, considerándolos como sujetos integrales, atravesados por distintas dimensiones y condiciones (en la que el ser estudiante es solo una de ellas).

Por otro lado, se puede plantear que los saberes que se enseñan en las escuelas, así como la manera en que estos son significados por quienes los aprenden, pueden constituirse como una vía para el sostenimiento de los jóvenes en las instituciones.

En efecto, resulta primordial que los contenidos que los jóvenes aprenden en las escuelas sean relevantes para sus vidas, que aporten herramientas para reflexionar sobre distintos acontecimientos que atraviesan, así como para decidir acerca del curso que tomarán sus trayectorias. Si esto sucede, se puede plantear que los saberes aprendidos en las escuelas se constituyeron como saberes socialmente productivos, definidos como "aquellos saberes que modifican a los sujetos enseñándoles a transformar la naturaleza y la cultura, modificando su habitus y enriqueciendo el capital cultural de la sociedad o la comunidad" (Puiggrós y Galiano, 2004, p. 13). 
Estos se distinguen de los conocimientos redundantes, orientados a demostrar el conocimiento material y cultural acumulado por la sociedad. Los saberes socialmente productivos, a diferencia de los redundantes, tienen la capacidad de reformar a los sujetos en tanto les enseñan a transformar la naturaleza y la cultura, es decir que procrean, generan, elaboran.

Además, estos saberes son históricos, es decir que no son socialmente productivos en sí mismos sino que varían según las épocas, los lugares y los procesos sociales. Por esto, distintos contextos históricos habilitan diferentes articulaciones de sentidos, produciendo que ciertos saberes se vuelvan socialmente productivos en un momento dado. Ahora bien, para que esto suceda, ese saber debe incidir en el proceso de construcción identitaria de los actores sociales involucrados, que a su vez deben reconocerlos como tales (Rodríguez, 2007).

En un proceso de escolarización, los saberes socialmente productivos pueden generarse a partir de distintas vivencias, como la formación en cierta temática que es relevante para quien la aprende, por los sentidos que genera o por los procesos que pone en movimiento. Claro que esto no puede ser definido a priori, sino que se vincula a la manera en que quienes participan los significan, y esto depende de la manera en que se planifican las actividades, de otras experiencias y saberes a los que se los liga, entre otras dimensiones.

Concretamente, en un contexto de complejización de los procesos de inserción laboral de jóvenes y de transformaciones del mercado de trabajo, la formación para el trabajo puede volverse un saber relevante para los jóvenes.

Esta formación puede suponer una capacitación en saberes generales para el trabajo, entre los que se destacan discutir en torno a la articulación entre educación y trabajo, sobre los saberes que circulan en el mundo productivo y cómo se aprenden, considerar las relaciones entre educación, ciencia y tecnología, producción y trabajo, ciudadanía y trabajo, así como también reflexionar críticamente acerca de las condiciones del mercado de trabajo, aspectos vinculados al derecho laboral y a la discriminación en el mundo del trabajo (Jacinto, 2013).

A esta formación amplia en saberes generales sobre el mundo del trabajo, se suma la implementación de prácticas específicas de formación para el trabajo, por ejemplo, la realización de pasantías o microemprendimientos, de procesos de orientación vocacional, etcétera (Jacinto, 2013).

En efecto, la preparación de los jóvenes para enfrentar un mercado laboral heterogéneo y segmentado requiere de una formación amplia que lo analice críticamente, que otorgue herramientas y que presente las reglas de juego que imperan en un campo en el que los jóvenes, si no se han incorporado paralelamente al cursado de sus estudios, lo harán de manera próxima, ya sea en relación de dependencia o de manera autogestiva, y resulta también valiosa la implementación de dispositivos de formación para el trabajo. En esta preparación, dada su masividad y su obligatoriedad, la escuela secundaria adquiere un papel central. 
Retomando lo expuesto, se puede plantear que la exclusión de la escuela y en la escuela son problemas distintos que suponen para su tratamiento estrategias distintas por parte del sistema educativo en general y de las instituciones en particular. Las primeras requieren el desarrollo de estrategias que se implementan para retener a los estudiantes en las escuelas, mientras que las segundas refieren a cómo lograr que los jóvenes realicen procesos educativos de calidad, que resulten significativos para sus vidas y les brinden herramientas para tomar decisiones y estructurar sus trayectorias.

En este sentido, mientras el seguimiento personalizado de los estudiantes se constituye en una estrategia de retención y por ello de inclusión a la escuela secundaria, la impartición de saberes socialmente productivos (y la formación para el trabajo podría conformarse como tales), deviene en una forma de incluir en la escuela.

A continuación, se presenta la propuesta educativa de una escuela secundaria de la ciudad de Neuquén, en la que la formación para el trabajo tiene un lugar central, para luego analizar los sentidos construidos por un grupo de egresados de la institución sobre sus procesos de escolarización.

\section{ESCUELA N: FORMACIÓN PARA EL TRABAJO COMO EJE DE LA PROPUESTA EDUCATIVA}

La Escuela $\mathrm{N}$ es una escuela secundaria pública de gestión privada ${ }^{7}$ que fue creada en 2005 en la ciudad de Neuquén. Otorga el título de Bachiller especializado en Economía Social y Prácticas de Emprendimientos, de manera conjunta con una Certificación para el Mundo del Trabajo que reconoce 1805 horas de capacitación en talleres de formación profesional, y que se distingue según el plan elegido: Diseños constructivos, Informática, Agropecuaria y Gastronomía. Tiene una matrícula de 260 estudiantes (mujeres y varones) de sectores socioeconómicos bajos.

La forma de gestión privada posibilita que el plantel docente sea seleccionado de manera directa por la institución (usualmente por su equipo directivo), y no por el sistema de puntaje docente en asambleas públicas del Consejo Provincial de Educación. Esta situación, en el caso analizado, facilitó el ingreso a la institución de profesores con perfiles militantes (políticos, sociales, religiosos), que imprimieron una lógica de trabajo e institucional específica que es, en parte, responsable del desarrollo de estrategias pedagógicas e institucionales que favorecen la inclusión educativa de los jóvenes.

\footnotetext{
${ }^{7}$ El hecho de que sea una institución pública de gestión privada significa que la responsabilidad de la gestión de los servicios educativos está a cargo de instituciones o personas particulares, en este caso el Obispado de la Provincia del Neuquén, a pesar de lo cual reciben aportes financieros por parte del Estado (especialmente para el pago de remuneraciones).
} 
Está ubicada en una zona semirrural de la ciudad, compuesta principalmente por asentamientos informales cuyos servicios de gas, agua y luz son deficitarios y las tenencias de la tierra son precarias. Se encuentra alejada del centro y el transporte público de pasajeros circula con poca frecuencia.

La formación para el trabajo atraviesa y otorga sentido a toda su propuesta educativa, y se construye desde los preceptos de la economía social. En este marco, se propone una visión alternativa a la hegemónica respecto de la sociedad y del mundo del trabajo y se considera al mercado laboral como un espacio complejo y precario, que no ofrece puestos de trabajo de calidad para todos, en el que los jóvenes (especialmente quienes provienen de sectores bajos) encuentran dificultades para insertarse en empleos, o lo hacen de manera inestable e informal. A partir de este diagnóstico se plantea la formación para la realización de emprendimientos productivos como eje de la propuesta educativa. Cabe aclarar que el trabajo autogestivo es planteado desde la perspectiva de la economía social, en la cual los componentes asociativos y cooperativos son claves, alejándose de la postura individualista propia de la ideología neoliberal. Desde esta lógica, el perfil de egresados que busca formar se condensa en la idea de emprendedor, que supone la formación de sujetos críticos y autónomos, capaces de reconocer situaciones de explotación laboral.

En su plan curricular se estipula que durante $1^{\circ}$ y $2^{\circ}$ año todos los estudiantes roten por los cuatro talleres y en $3^{\circ}$ elijan uno de ellos, en el que se especialicen hasta $5^{\circ}$ año. A partir de $3^{\circ}$ año, se imparten materias específicas de la orientación en las que se acompaña la elaboración de un emprendimiento productivo (Teoría de microemprendimiento, Práctica de microemprendimiento, Contabilidad y Economía Social), ya que durante ese año los estudiantes comienzan a planificar y escribir el proyecto de microemprendimiento que ejecutarán durante $4^{\circ}$ y $5^{\circ}$ año.

Desarrollan distintas prácticas de formación para el trabajo: en primer lugar, talleres de formación profesional en el marco de los cuales se realizan emprendimientos productivos orientados a desarrollar la autonomía y el cooperativismo en la inserción laboral de los jóvenes; en segundo lugar, procesos de orientación vocacional y ocupacional, que comienzan en $4^{\circ}$ año y finalizan en $5^{\circ}$, en los que buscan que los jóvenes reflexionen sobre sus intereses ocupacionales y de formación; en tercer lugar, pasantías que se implementan como corolario del proceso de orientación vocacional, cuyo objetivo es que los estudiantes tengan un acercamiento a la realidad laboral local; en cuarto lugar, cursos especiales orientados al mundo del trabajo desarrollados durante tres semanas al año en las que se suspenden las clases y se imparten cursos acotados en el marco de cada especialidad; por último, en el marco de las orientaciones, experiencias reales de trabajo como servicios de cáterin. Entonces, se puede plantear que la formación para el trabajo propuesta implica una capacitación amplia en saberes generales del mundo laboral, así como prácticas específicas de formación para el trabajo (Jacinto, 2013), desarrollando una propuesta de formación integral. 
Finalmente, desarrollan distintas estrategias orientadas al sostenimiento de los jóvenes en la escuela. En efecto, el seguimiento personalizado de los estudiantes tiene un papel central y se estructura de dos maneras distintas. El primer grupo de prácticas refiere a las relaciones de proximidad entre los adultos de la escuela y los estudiantes, que se manifiesta en el conocimiento que docentes y directivos suelen tener de las historias personales, laborales y familiares de los jóvenes, así como de sus trayectorias escolares. Tal como planteaba Nóbile (2011) desde la teoría, esto es observado como un aspecto fundamental en las estrategias para alcanzar la inclusión educativa de los alumnos, en las que las relaciones de confianza y proximidad operan como un factor que les ayuda a sostenerse dentro de la institución. Ejemplo de esto es el seguimiento desde la escuela cuando un joven se ausenta en reiteradas oportunidades, que implica notificar a sus familias acerca de que este no va a la institución, conocer las causas de las inasistencias y eventualmente intervenir para que continuaran sus procesos de escolarización.

Otro grupo de prácticas que buscan sostener a los jóvenes dentro de la escuela son las estrategias pedagógicas orientadas a que los estudiantes promuevan las asignaturas, que favorecen la retención (Montes y Ziegler, 2010). Se destacan la implementación de profesores ayudantes en algunas materias, la realización de clases de apoyo antes del cierre de cada trimestre (se suspenden las clases y, mientras quienes están al día con las materias asisten a talleres artísticos y orientados al mundo del trabajo, los docentes trabajan de manera personalizada con quienes presentan dificultades para aprobar el trimestre) y la constitución de mesas de exámenes extraordinarias para estudiantes de $5^{\circ}$ año.

En el apartado siguiente, se analizarán los sentidos que egresados de esta escuela habían construido sobre el pasaje por la institución y la formación recibida, para pensar la manera en que distintos elementos y estrategias se constituían como factores favorecedores de su inclusión a la escuela y en la escuela.

\section{DESARROLLANDO PROCESOS EDUCATIVOS SIGNIFICATIVOS. VALORACIONES DE EGRESADOS SOBRE SUS EXPERIENCIAS ESCOLARES}

A partir del seguimiento de la cohorte de egresados 2009 de la escuela, de manera sintética se puede sostener que para los jóvenes entrevistados haber asistido a esta institución había sido significativo por distintos motivos, que agrupamos en dos ejes: por un lado, la valoración del seguimiento personalizado; por el otro, la valoración de los saberes aprendidos y de la formación para el trabajo recibida. A su vez, las estrategias, dinámicas y saberes impartidos habían resultado maneras de incluir a los estudiantes a la escuela y en la escuela. A continuación, desarrollaremos cada uno de ellos. 


\section{Eje 1. Valoración del seguimiento personalizado}

El primer eje de la valoración se vincula al seguimiento personalizado que habían tenido los jóvenes, que se concretaba a partir de las relaciones de proximidad entabladas con los docentes y directivos de la escuela, así como desde distintas propuestas pedagógicas que implicaban el desarrollo de mayores oportunidades para comprender y aprender contenidos, y así aprobar las materias. Ambas dinámicas eran identificadas como aspectos facilitadores de sus procesos de escolarización, y en este sentido, se constituían en estrategias que habían evitado la exclusión de la institución (Gluz, 2016).

Respecto del primer eje, el de las relaciones de proximidad, rescataban que les habían ayudado a resolver situaciones personales complejas y los habían contenido en momentos difíciles:

Eran comprensivos, tenían paciencia. Casi todos nos ayudaban. Cuando faltaban chicos a la escuela los salían a buscar, a ver por qué faltaban (Lucía, comunicación personal, 2014).

En la escuela era todo más familiar. Cualquiera de ellos [docentes o directivos] te preguntaba si tenías algún problema o algo y siempre estaban ahí para ayudarte. Eso es re importante de la escuela y me ayudó un montón (Natalia, comunicación personal, 2014).

En este sentido, valoraban el hecho de encontrar en los docentes, personas con quienes dialogar y buscar ayuda en caso de situaciones personales problemáticas:

Nos influenciaban en cómo estás vos, cómo está tu familia, te sentís bien, qué necesitas, te puedo ayudar, vamos a hacer algo para que te sientas mejor, vamos a ver cómo podemos solucionar esto, vamos a buscarle la vuelta para progresar, para salir adelante. Si no podemos así, vamos a intentar así. (...) En otra escuela vas, te sentás en el aula y sos uno más. Para mí en esta escuela no (Fernanda, comunicación personal, 2014).

Pero yo me llevo más que nada lo humano, la clase de personas que conocí ahí adentro, (...) los profes, los estudiantes, hasta los porteros, con todo el mundo eran muy cálidas las relaciones que había ahí adentro. Era más que nada lo humano, a pesar de todos los cursos, capacitaciones que hicimos. (...) Nunca te dejaban salirte, caerte (Nadia, comunicación personal, 2014).

Sabíamos que si tenías algún problema, venías acá y te sentabas y charlabas. Te sabían entender, te ayudaban. (...) Valoraban tu persona, a vos qué te pasaba. Una contención. (...) hay veces que necesitás que te pregunten, que no es por rebelde no más (Graciela, comunicación personal, 2014). 
Se pone de manifiesto que, desde la perspectiva de los egresados, los factores extraescolares, antaño excluidos de los procesos escolares, ingresaban a las instituciones. Pero además, los jóvenes destacaban que no solamente los escuchaban (lo cual de por sí era valorado), sino que también desde la escuela se los acompañaba e incluso intervenía para que los problemas personales que tenían no se convirtieran en causales de abandono de sus procesos educativos.

Además, en la valoración que los estudiantes hacían respecto del seguimiento personalizado que recibían, quedaba a la vista también el compromiso docente orientado a sostener a los estudiantes dentro de las aulas (Nóbile, 2011):

Con tantas cosas que pasé en mi casa, tenía la contención [en la escuela]. (...) Como que los profesores y todos los que estaban ahí, cuando yo estaba mal, me ayudaron a hacerme responsable de mí y de todos mis hermanos y de mi casa. (...) Siempre la escuela se dedicó no a echar sino a contener (Waldo, comunicación personal, 2014).

El segundo eje del seguimiento personalizado de los estudiantes se observaba en la organización de estrategias pedagógicas de apoyo a las trayectorias educativas (Montes y Ziegler, 2010). En los distintos espacios orientados a este fin, los estudiantes tenían la posibilidad de revisar contenidos y saberes que no habían podido aprender en los tiempos pautados y de esta manera evitar el retraso. Estas intervenciones también eran valoradas por los estudiantes:

Me gustó por parte de los profesores, la contención que tenían hacia nosotros y la dedicación para que nosotros estudiemos (Leandro, comunicación personal, 2014).

A mí me gustó el colegio porque si había chicos que dejaban de estudiar ellos trataban de ver la situación, de ver qué era lo que pasaba, por qué no iba a estudiar. Nos daban la posibilidad de rendir cuando quedábamos libres. Yo en $2^{\circ}$ año había quedado libre y se armó un grupo de los chicos que habíamos quedado libres para rendir las materias. Nos juntábamos, estudiábamos, el que entendía una materia les explicaba a los otros. Nos juntábamos, íbamos estudiando y sacando las materias, se nos hacía más fácil. Eso fue idea de los profes. Ellos veían mucho la situación de nosotros, nos daban muchas posibilidades para estudiar. (...) Era como que se preocupaban bastante, estaban todo el tiempo con nosotros (Natalia, comunicación personal, 2014).

De esta manera, el seguimiento personalizado (tanto por la confianza en las relaciones interpersonales y la proximidad de los vínculos entre docentes y estudiantes, como por la generación de estrategias pedagógicas de apoyo a las trayectorias educativas), se constituía en un aspecto valorado por los estudiantes y como uno de los factores que favorecía el sostenimiento de los jóvenes en la escuela, propiciando su inclusión a la escuela. 


\section{Eje 2. Valoración de la formación para el trabajo y de los saberes aprendidos en la escuela}

El segundo grupo de valoraciones refiere a los distintos tipos de saberes (técnicos, socioemocionales, relacionales, etcétera) aprendidos en la escuela y a la formación para el trabajo recibida a partir del pasaje por prácticas de formación para el trabajo (como talleres, pasantías, orientación vocacional, emprendimientos productivos, etcétera), así como de otros espacios curriculares. Esta valoración tenía distintas aristas.

Un primer aspecto refería a la transmisión de saberes, específicamente la formación en saberes técnicos de las orientaciones que habían cursado:

Chacinados, y todo lo que es marcar a los chanchos (...) Todo hacíamos nosotros. Íbamos allá arriba de la meseta, nos llevaban y a los animales de los vecinos los desangrábamos, le sacábamos sangre para llevar muestras a Zoonosis y esas cosas (Emilio, comunicación personal, 2014).

Me gustó mucho, fue muy enriquecedora porque fue aprender y valorar [lo que se hace] para que sea puesta en la mesa del consumidor, todos los detalles, las cocciones, que tenga buen piso, porque a veces vas a comer una pizza a un lugar y se cae. Cosas, detalles que me gustaron (Leandro, comunicación personal, 2014).

Otro tipo de saberes que rescataban eran aquellos necesarios para montar un emprendimiento productivo, tales como la planificación, la redacción del proyecto, la puesta en marcha o ejecución, la evaluación:

Aprendí esto de vender el producto que hacemos, poder sacar los costos bien, que haya ganancia en cada cosa que hacíamos... y que también el producto salga bien (Gabriela, comunicación personal, 2014).

Habíamos tomado responsabilidades bastante importantes. Manejábamos la plata nosotros, íbamos a comprar, a las imprentas, pedíamos presupuestos. (...) Y en el taller aprendimos un montón de lo que tiene que ver con el oficio, no solamente de lo técnico sino de cómo uno tiene que trabajar para hacer un emprendimiento. [Aprendimos] esto de entender que un emprendimiento es un proyecto propio que se tiene que sustentar con mucho trabajo y que implica una relación horizontal entre las partes (Irene, comunicación personal, 2014).

Referido a la panadería hacíamos recetas, teníamos que aprender a sacar costos para después venderlos (...). Todo lo que hacíamos era para cuando nosotros termináramos de estudiar, para tener nuestro emprendimiento. También nos enseñaron a sacar costos para vender el producto (Verónica, comunicación personal, 2014). 
De la realización de los emprendimientos productivos también destacaban el aprendizaje de los contenidos específicos de cada proyecto en particular:

Aprendí todo lo relacionado con el emprendimiento en sí, que eran los animales, los chanchos, cómo tener en condiciones el chiquero... Qué tipo de corral convenía usar, qué materiales, cómo teníamos que cuidar a la chancha, qué vacunas, qué alimentos les teníamos que dar y cómo, qué cantidad. Un montón de cosas, (...) porque tenés que darte cuenta cuándo la chancha está enferma, cuándo no, cuándo está en su peso ideal, cuándo no, si está flaca. Y después llevarlo al matadero, que lo maten, a un matadero que esté certificado o algo así (Fernanda, comunicación personal, 2014).

Me aportó a nivel conocimiento al máximo, Corel, Photoshop, Word, Excel, Power Point. Todos los programas, que incluso son los que hoy utilizo en el trabajo, para lo que sea. (...) Cuando teníamos una muestra también tenías relación social, tenías que aprender a venderte, hacías un poquito de atención al público. Nos enseñaron de todo un poco, era un emprendimiento netamente (Esteban, comunicación personal, 2014).

Otro tipo de saberes que rescataban los jóvenes eran de tipo relacional, y referían a la formación en disposiciones personales tales como la responsabilidad, el trabajo en equipo, el compañerismo:

Sí, estuvo lindo porque aprendés mucho y también está esto de la responsabilidad de vender, esto de ser más responsables. Y no sólo para ese trabajo sino para otras cosas, acá en la Universidad también (...). Eso te ayuda a ser responsable porque vos, ponele tenías encargos y los tenías que hacer, o sabías que tenías que pagar esto de la luz o las materias primas y tenías que vender para pagarlos. (...) También te sirve para desenvolverte en un trabajo que tengas más adelante o cuando yo me reciba. El ser responsable, el saber que tenés que ir a un horario, y vos tenías (...) esa hora para hacer una receta o tenías que hacer lo que ibas a vender al otro día (Noelia, comunicación personal, 2014).

También valoraban las habilidades comunicacionales que la participación en distintas prácticas de formación para el trabajo les había ayudado a desarrollar:

Aprendí la parte de costos, la paciencia, cómo hablar, la oratoria, el léxico. No se puede hablar de la misma manera a la doctora sobre un emprendimiento que para una emprendedora que viva en una toma o en mi barrio (Esteban, comunicación personal, 2014).

Además, se ponía de manifiesto la autonomía que habían adquirido los jóvenes a partir de la formación recibida, que aparecía en el hecho de proyectar inserciones laborales de manera independiente, de tener distintos tipos de proyectos a futuro (no sólo en el plano laboral), etcétera: 
La idea se me empezó a dar, esto de tener mi propia fuente de trabajo, cuando [la profesora] empezó a darnos el taller de economía social y microemprendimientos. Ahí hablábamos todo de cómo se maneja un emprendedor, cómo arranca, que tiene que llevar una contabilidad, todas las cosas que tiene que tener un emprendedor. (...) Yo creo que eso me hizo dar un giro en la cabecita y decir, puede ser que uno puede llegar a ser su propio patrón. Está re bueno esto, es otra cosa (Lucas, comunicación personal, 2014).

Ellos te preparan para que tengas tu salida laboral, pero más que nada para que vos seas un emprendedor, para que vos tengas salida pero que la armes vos tu salida laboral. Ahora yo soy dependiente, pero te preparan para que vos tengas tu propio trabajo y que vos seas tu propio jefe, y me gustaría que así sea (Nadia, comunicación personal, 2014).

Después de que me reciba, voy a tratar de trabajar como docente, hasta que el día de mañana pueda lograr mi proyecto de futuro que es armar un gimnasio, no un gimnasio de gran costo sino un gimnasio más o menos económico donde pueda asistir mucha gente (Emilio, comunicación personal, 2014).

La formación recibida además era valorada porque había incidido en el curso de sus trayectorias educativo-laborales de distintas maneras. En primer lugar, en los casos de los jóvenes que habían continuado estudiando en la universidad, les había ayudado a definir qué carrera universitaria estudiar, o les había brindado información sobre los planes de estudio, etcétera:

Fue bueno porque me ayudó mucho, más allá de que hablé con el profesor de educación física y él me ayudó a entusiasmarme, también iba viendo las materias que teníamos en el profesorado. (...) Me ayudó a decir lo principal es terminar esta carrera [Profesorado en Educación Física] para después hacer lo que yo en realidad quería hacer que era veterinaria. Pero me ayudó para meterme enseguida y no dejar pasar el tiempo y estudiar más tarde (Emilio, comunicación personal, 2014).

Hasta $5^{\circ}$ año como que no sabía nada seguro. (...) Me obligaban mis viejos a estudiar.... Era una presión constante no saber qué estudiar.... Y la escuela me ayudó en ese sentido, sobre todo en el último año cuando hicimos un curso de orientación vocacional (...), además nos hacían entrevistas para ver si nos gustaba lo del taller, qué tipo de empleos nos gustaban. (...) La orientación era cómo emprender y a insertarnos en el mundo laboral, nos enseñaban el currículum, la forma de presentación, como armar el discurso (Irene, comunicación personal, 2014).

Me ayudó a elegir una carrera que me guste, porque también hacían preguntas referidas a las carreras, la relación que teníamos con esa profesión que queríamos elegir (Gabriela, comunicación personal, 2014). 
En segundo lugar, la escuela había intervenido en sus procesos de inserción laboral, como puente con el mundo del trabajo. Tal había sido el caso de algunos jóvenes que habían continuado trabajando en las unidades productivas en las que habían realizado las pasantías, o de docentes de la institución que habían establecido contactos entre estudiantes y empleadores, ampliando la red de relaciones de los jóvenes:

Empecé en agosto creo de $5^{\circ}$ año y después cuando terminé el secundario, estaba trabajando ahí. Ya había terminado mi pasantía, pero ellos me dijeron que si yo me quería quedar trabajando, que me quedara trabajando, como yo quisiera. Y yo sí, me re gustaba laburar con ellos (Rocío, comunicación personal, 2014).

La pasantía comenzó en octubre más o menos y hasta diciembre que me egresé y después seguí (Leandro, comunicación personal, 2014).

En tercer lugar, otra manera en que la escuela había intervenido en el curso de las trayectorias laborales juveniles había sido a partir de haberles brindado herramientas para que montaran un emprendimiento productivo:

Si yo no hubiera tenido una introducción de lo que se trataba ser un emprendedor, no hubiera emprendido un proyecto, algo propio. No hubiera tenido la idea de qué actitud tomar. A mí la idea que me llevó a tener un proyecto, un emprendimiento mío fueron esas clases (Lucas, comunicación personal, 2014).

No hacía gastronomía pero sí otro tipo de emprendimiento, que está relacionado. En esto de administrar, de saber que tenés que comprar pero también tenés que pagar lo que usas. La planificación de lo que es tu trabajo. Eso sí, eso lo vi en Gastronomía y de ahí me quedó y lo uso (Noelia, comunicación personal, 2014).

Finalmente, en algunos casos, la formación técnica recibida había estructurado trayectorias laborales en los oficios aprendidos en la escuela:

Yo aprendí electricidad en el colegio, lo básico, lo monofásico. Aprendí en el colegio y lo usé siempre, porque los profesores nos enseñaban a trabajar en realidad, porque cuando a uno le enseñan, le enseñan a trabajar con guantes, las herramientas que tenías que usar, si vas a pelar un cable, sabés que está el alicate, y todas esas cosas las usás (Lucas, comunicación personal, 2014).

Si bien el peso de los factores estructurales (como las condiciones del mercado de trabajo) continuaban limitando la potencia de esta formación, los jóvenes valoraban el formarse en distintos saberes (técnicos, para emprender, relacionales, comunicacionales) y planteaban que les habían brindado herramientas útiles para orientar sus trayectorias posteriores (cursando estudios superiores, montando emprendimientos productivos, trabajando en oficios aprendidos, etcétera). 
Por este motivo, se puede postular que el pasaje de estos jóvenes por la institución no habían sido procesos de escolarización de baja intensidad (Kessler, 2004) o meros "estar en el aula", sino que la formación recibida era valorada por su incidencia en la construcción de la subjetividad de este grupo de egresados, de manera tal que los saberes impartidos en la escuela se habían constituido como saberes socialmente productivos (Puiggrós y Galiano, 2004), favoreciendo el desarrollo de procesos educativos significativos y por ello la inclusión en la escuela (Gluz, 2016).

\section{CONCLUSIONES}

A partir de lo trabajado en el artículo se pone de manifiesto que los egresados destacaban distintos aspectos de sus pasos por la institución educativa como importantes para sus vidas. En primer lugar, valoraban el seguimiento personalizado que habían tenido, tanto por medio de dispositivos de apoyo a las trayectorias educativas como por la cercanía de los vínculos con docentes y directivos y la contención recibida. En segundo lugar, referían a la formación recibida (en los oficios específicos, en disposiciones personales, en aprendizajes para la realización de emprendimientos productivos, entre otros). En tercer lugar, valoraban la intermediación en sus búsquedas laborales o procesos de inserción (tanto en relación de dependencia como de manera independiente). Finalmente, destacaban que les habían brindado información para ayudarles a definir qué carreras universitarias continuar estudiando. En este sentido, se constataba la valoración de la formación para el trabajo recibida, tanto desde una perspectiva amplia como desde una perspectiva específica, referida al aprendizaje de un oficio.

Pero, además, las dinámicas que resultaban en un seguimiento personalizado de los estudiantes (relaciones de proximidad y contención, así como dispositivos pedagógicos de apoyo a las trayectorias educativas) eran rescatadas por los jóvenes como facilitadores de sus procesos educativos, de manera tal que promovían la inclusión de los jóvenes a la escuela secundaria.

Por otro lado, la relevancia que los jóvenes asignaban a los saberes promovidos desde la institución, y a las prácticas de formación para el trabajo impulsadas en las escuelas, daban cuenta de la importancia que tenían estos saberes y estas prácticas en la vida de los jóvenes, ya que eran rescatados como importantes para la toma de decisiones que afectaban el curso de sus trayectorias posteriores.

Así, haber asistido a esta escuela no había sido una mera formalidad para la obtención de la credencial educativa: en tanto les habían brindado herramientas para analizar, comprender y transformar la propia realidad, mostraban su capacidad creadora, por lo cual se volvían, a la luz de la valoración y de la incidencia en el curso de vida de los jóvenes, y más aún en contextos de dificultades para finalizar el nivel medio e insertarse laboralmente, saberes socialmente productivos. 
En efecto, de los relatos presentados se desprende que haber asistido a esta escuela había sido significativo en términos subjetivos para los egresados. La valoración que los jóvenes realizaban acerca de sus procesos de escolarización daba cuenta de la incidencia en la construcción de las identidades de los jóvenes.

Por todo esto, se puede plantear que, en un contexto de exclusión de la escuela secundaria y en la escuela secundaria, los saberes y las prácticas pedagógicas propuestos por la Escuela N, especialmente aquellos vinculados a la formación para el mundo del trabajo, favorecieron la retención de estos jóvenes en las aulas y con ello la terminalidad del nivel, así como la construcción de saberes valorados por ellos mismos como relevantes para sus trayectorias vitales. De esta manera, la propuesta educativa de la Escuela $\mathrm{N}$ había habilitado la inclusión a la escuela secundaria y en la escuela secundaria de estos jóvenes.

Finalmente, queda claro que estos análisis corresponden a aquellos jóvenes que habían logrado sostenerse y culminar sus estudios secundarios. Cabe preguntarse, como interrogante para responder en futuras líneas de indagación, qué sentidos habrían construido respecto de sus procesos educativos, así como la incidencia de la formación recibida en las trayectorias vitales posteriores de aquellos jóvenes que abandonaron sus estudios sin haber obtenido la credencial del nivel secundario.

\section{REFERENCIAS}

Arriagada, C., y López, D. (2015). La Educación Secundaria en Chile: Situación actual y proyecciones. Praxis educativa, 19(2), 13-18.

Bertranau, F., y Casanova, L. (2014). Informalidad laboral en la Argentina: segmentos críticos y políticas para la formalización. Buenos Aires, Argentina: Organización Internacional del Trabajo.

Bourdieu, P. (1988). La distinción. Criterio y bases sociales del buen gusto. Madrid, España: Taurus.

Cabrera Borges, C. (2017). ¿Cómo retener a los jóvenes y adultos en el sistema educativo público? Análisis de las estrategias desplegadas en tres liceos nocturnos del Uruguay. Páginas de Educación, 10(2), 57-78. https://doi.org/10.22235/pe.v10i2.1424

Camillioni, A. (2006). El saber sobre el trabajo en el currículo escolar. Anales de la educación común, 2(3), 112-117.

Croso, C. (2009). Universalizar el acceso y completar la educación secundaria. Entre la meta social y la realidad latinoamericana. Buenos Aires, Argentina: SITEAL/IIPE-UNESCO Sede Regional Buenos Aires/OEl.

Consejo Federal de Educación. (2009). Orientaciones para la organización pedagógica e institucional de la educación secundaria obligatoria. Recuperado de https://www.argentina.gob.ar/sites/default/files/93-09-anexo-5900b6d3bab8e.pdf 
Dussel, I. (2009). La escuela media y la producción de la desigualdad: continuidades y rupturas. En G. Tiramonti y N. Montes (Comps.), La escuela media en debate. Problemas actuales y perspectivas desde la investigación (pp. 39-52). Buenos Aires, Argentina: Manantial/FLACSO.

Gentili, P. (2011). Pedagogía de la igualdad. Ensayos contra la educación excluyente. Buenos Aires, Argentina: Siglo XXI.

Gluz, N. (2016). Políticas y prácticas en torno a la "inclusión escolar". ¿Por qué es tan difícil la democratización escolar? Buenos Aires, Argentina: Estación Mandioca.

Grupo Viernes. (2008). Una experiencia de cambio en el formato de la escuela media: las Escuelas de Reingreso en la Ciudad de Buenos Aires. Revista Propuesta Educativa, 17(30), 57-69.

Jacinto, C. (2013). La formación para el trabajo en la escuela secundaria como reflexión crítica y como recurso. Revista Propuesta Educativa, 22(40), 48-63.

Kessler, G. (2004). Sociología del delito amateur. Buenos Aires, Argentina: Paidós.

Mallimaci, F., y Giménez Béliveau, V. (2006). Historia de vida y métodos biográficos. En I. Vasilachis de Gialdino (Coord.), Estrategias de investigación cualitativa (pp. 175-212). Barcelona, España: Gedisa.

Montes, N., y Ziegler, S. (2010). Miradas sobre una experiencia de cambio en la escuela secundaria. Nuevos formatos para promover la inclusión educativa. Revista Mexicana de Investigación Educativa, 47(15), 1075-1092.

Nóbile, M. (2011). Redefiniciones de la relación docente-alumno: una estrategia de personalización de los vínculos. En G. Tiramonti (Dir.), Variaciones sobre la forma escolar. Límites y posibilidades de la escuela media (pp. 179-204). Rosario, Argentina: Homo Sapiens Ediciones/Buenos Aires, Argentina: FLACSO.

Organización Internacional del Trabajo. (2013). Trabajo decente y juventud en América Latina. Lima, Perú: Organización Internacional del Trabajo.

Pérez, P. (2008). La inserción ocupacional de los jóvenes en un contexto de desempleo masivo. Buenos Aires, Argentina: Miño y Dávila.

Puiggrós, A., y Gagliano, R. (Dir.). (2004). La fábrica del conocimiento. Los saberes socialmente productivos en América Latina. Rosario, Argentina: APPEAL/Homo Sapiens.

Rodríguez, L. M. (2007). Saberes socialmente productivos, formación y proyecto. En M. Gómez Sollano (Coord.), Saberes socialmente productivos y educación. Contribuciones al debate (pp. 55-60). México D. F., México: Universidad Nacional Autónoma de México.

Sautu, R. (1999). El método biográfico. La reconstrucción de la sociedad a partir del testimonio de los actores. Buenos Aires, Argentina: Editorial Belgrano.

Southwell, M. (2011). La educación secundaria en la Argentina. Notas sobre la historia de un formato. En G. Tiramonti (Dir.), Variaciones sobre la forma escolar. Límites y posibilidades de la escuela media (pp. 35-70). Rosario, Argentina: Homo Sapiens Ediciones/Buenos Aires, Argentina: FLACSO. 
Tenti Fanfani, E. (2007). La escuela y la cuestión social. Ensayos de sociología de la educación. Buenos Aires, Argentina: Siglo XXI.

Terigi, F. (2007, mayo). Los desafíos que plantean las trayectorias escolares. Ponencia presentada en el III Foro Latinoamericano de Educación. Jóvenes y docentes. La escuela secundaria en el mundo de hoy, Buenos Aires, Argentina.

Tiramonti, G. (2011). Escuela media: la identidad forzada. En G. Tiramonti (Dir.), Variaciones sobre la forma escolar. Límites y posibilidades de la escuela media (pp. 17-34). Rosario, Argentina: Homo Sapiens Ediciones/Buenos Aires, Argentina: FLACSO. 